\title{
COMMUTATIVITY THEOREMS FOR RINGS WITH CONSTRAINTS ON COMMUTATORS
}

\author{
HAMZA A.S. ABUJABAL \\ Department of Mathematics \\ Faculty of Science \\ King Abdul Aziz University \\ P. O. Box 31464, Jeddah 21497 \\ Saudi Arabia
}

(Received August 29, 1989 and in revised form April 19, 1991)

\begin{abstract}
In this paper, we generalize some well-known commutativity theorems for associative rings as follows: Let $n>1, m, n$, and $t$ be fixed non-negative integers such that $s \neq m-1$, or $t \neq n-1$, and let $R$ be a ring with unity 1 satisfying the polynomial identity $y^{s}\left[x^{n}, y\right]=\left[x, y^{m}\right] x^{t}$ for all ; $y \in R$. Suppose that (i) $R$ has $Q(u)$ (that is $n[x, y]=0$ implies $[x, y]=0$ ); (ii) the set of all nilpotent clements of $R$ is central for $t>0$, and (iii) the set of all zero-divisors of $R$ is also central for $t>0$. Then $R$ is commutative. If $Q(n)$ is replaced by " $m$ and $n$ are relatively prime positive integens," then $R$ is commutative if extra constraint is given. Other related commutativity results are also obtained.
\end{abstract}

KEY WORDS AND PHRASES. Commutativity of rings, Torsion free rings, Ring with unity, Semi-prime Rings.

1980 AMS SUBJECT CLASSIFICATION CODE. 16 A70.

\section{INTRODUCTION.}

Throughout this paper, $R$ will be an associative ring (may be without unity 1 ). $Z(R)$ will represent the center of $R, N(R)$ the sct of all nilpotent elements of $R, N^{\prime}(R)$ the set of all zero-divisors in $R$ and $C^{\prime}(R)$ the commutator ideal of $R$. For any $x, y \in R$, we write as usual $[x, y]=x y-y x$. By $G F(q)$, we mean the Galois field (finite field) with $q$ elements, and $(G F(q))_{2}$ the ring of all $2 \times 2$ matrices over $G F(q)$. Set $e_{11}=\left(\begin{array}{ll}1 & 0 \\ 0 & 0\end{array}\right), e_{12}=\left(\begin{array}{ll}0 & 1 \\ 0 & 0\end{array}\right)$, $e_{21}=\left(\begin{array}{ll}0 & 0 \\ 1 & 0\end{array}\right)$, and $e_{22}=\left(\begin{array}{ll}0 & 0 \\ 0 & 1\end{array}\right)$ in $(G F(p))_{2}$ for a prime $p$.

For any positive integer $n$, the ring $R$ is said to have property $Q(n)$ if for all $x, y \in R$, $n[x, y]=0$ implies $[x, y]=0$. The property $Q(n)$ is an $H$-property in the sense of [1]. It is obvious that every $n$-torsion free ring $R$ has the property $Q(n)$, and every ring has the property $Q(1)$. Also, it is clear that if a ring $R$ has the property $Q(n)$, then $R$ has the property $Q(m)$ for every divisor $m$ of $n$.

Let $n>1, m, s$, and $t$ be fixed non-negative integers. The objective of this paper is to investigate the commutativity of a ring $R$ satisfying the polynonial identity

$$
y^{s}\left[x^{n}, y\right]=\left[x, y^{m}\right] x^{t} \text { for all } x, y \in R .
$$

To establish the commutativity of a ring $R$ with unity 1 satisfying the polynomial identity (1.1), we need some additional constraints. They frequently concern the torsion freeness of the commutators. But in fact, this constraint is not enough to prove the commutativity of the above mentioned ring. This could be solved by imposing further restrictions on $s$, and $t$. An example is also given to justify the constraints. 
Our results generalize some well-known commutativity theorems, for instance the result of Abu-Khuzam et al [2], Bell [3], and Psomopoulos ot al [4].

\section{PRELIMINARIES.}

In preparation for the proof of our results, we first state the following well-known lemmas.

LEMMA 1 ([5, Lemma 3]). Let $x$ and $y$ be clements in a ring $R$. If $[[x, y], x]=0$, then for any positive integer $k$,

$$
\left[x^{k}, y\right]=h \cdot x^{h-1}[x, y] \text {. }
$$

LEMMA 2 ([5, Lemma 4]). Let $R$ be a ring with unity 1 . and let $f: R \rightarrow R$ be a function such that $f(x+1)=f(.1)$ holds for crely $x \in R$. If for some positive integer $k$, $x^{k} f(x)=0$ or $f(x) x^{k}=0$, for every $x \in R$, then necessarnly $f(x)=0$.

LEMMA 3 ([6, Lemma 3$]$ ). Let $R$ be a ring with unity 1 , and let $x$ and $y$ be elements in $R$. If $\left(1-y^{k}\right) x=0$, then $\left(1-y^{k m}\right) x=0$ for any integers $k>0$ and $m>0$.

LEMMA 4 ([7, Theorem]). Let $f$ be a polynomial in $n$ non-commuting indeterminates $x_{1}, x_{2}, \ldots, x_{n}$ with relatively prime integral coefficients. Then the following are equivalent:

(1) For any ring satisfying the polynomial identity $f=0, C(R)$ is a nil ideal.

(2) For every prime $p,(G F(p))_{2}$ fails to satisfy $f=0$.

(3) Every semi-prime ring satisfying $f=0$ is commutative.

LEMMA 5 ([8, Lenma 4]). Let $R$ be a ring with unity 1 , and let $x \in R$. If for each $y \in R$ there exist relatively prime positive integer's "I and $n$ such that $\left[x, y^{m}\right]=0$ and $\left[x, y^{n}\right]=0$, then $x$ is in the center of $R$.

LEMMA 6. Let $x$ and $y$ be clements in a ring $R$. Suppose that there exist relatively prime positive integers $m$ and $n$ such that $m[x, y]=0$ and $n[x, y]=0$. Then $[x, y]=0$.

THEOREM H. ([9, Theorem 18]). Let $n>1$ be a fixed integer. If $R$ is a ring with $x^{n}-x \in Z(R)$ for all $x \in R$, then $R$ is commutative.

\section{RESULTS.}

If $R$ satisfies the polynomial identity (1.1), then ly repeated use of (1.1), we have $y^{m s+s}\left[x^{n^{2}}, y\right]=\left(y^{m}\right)^{s}\left[x^{n}, y^{m}\right]\left(x^{n}\right)^{t}=\left[r, y^{m^{2}}\right], x^{\prime \prime t+t}$. Let $s^{\prime}=m s+s$, and $t^{\prime}=n t+t$. Then

$$
y^{s^{\prime}}\left[x^{n^{2}}, y\right]=\left[x, y^{m^{2}}\right] \cdot x^{t^{\prime}} \text { for all } x, y \in R .
$$

LEMMA 7. Let $n>1, m, s$, and $t$ be fixed non-negative integers, and let $R$ be a ring satisfying the polynomial identity (1.1), then

$$
C(R) \subseteq N(R)
$$

PROOF. If $m=0$, then $x=\epsilon_{11}$, and $y=e_{21}+\epsilon_{22}$ in $(G F(p))_{2}$, for a prime $p$, fail to satisfy $y^{s}\left[x^{n}, y\right]=0$. Further, if $m \geq 1$, then $x=e_{12}$, and $y=e_{11}$ in $(G F(p))_{2}$, for a prime $p$, fail to satisfy the polynomial identity (1.1). By Lemma 4. (3.2) holds.

Combining Lemma 4 with Lemma 7 gives the following commutativity theorem for semiprime rings.

THEOREM 1. Let $n>1, m, s$, and $t$ be fixed non-negative integers. If $R$ is a semiprime ring satisfying the polynomial identity (1.1), then $R$ is commutative.

LEMMA 8. Let $n>1, m$, and $s$ be fixed non-negative integers, and let $R$ be a ring with unity 1 satisfying the polynomial identity

$$
y^{s}\left[x^{n}, y\right]=\left[x, y^{m}\right] \text { for all } x, y \in R .
$$

If, further, $R$ has $Q(n)$, then

$$
N(R) \subseteq Z(R) .
$$

PROOF. Let $a \in N(R)$. Then there exists a minimal positive integer $p$ such that

$$
a^{\ell} \in Z(R) \text { for all } k \geq p .
$$

If $p=1$, then $a \in Z(R)$. Now, suppose $p>1$. Replace $x$ by $a^{p-1}$ in $(3.3)$ to get $y^{s}\left[\left(a^{p-1}\right)^{n}, y\right]=$ $\left[a^{p-1}, y^{m}\right]$. In view of $(3.5)$, and $(p-1) n \geq p$, the last polynomial identity implies that 


$$
\left[a^{\prime-1}, y^{\prime \prime \prime}\right]=0 \text { for all } y \in R
$$

Next, replace $x$ by $\left(1+a^{p-1}\right)$ in $(3.3)$ to obtain $y^{*}\left[\left(1+a^{p-1}\right)^{n}, y\right]=\left[1+a^{p-1}, y^{m}\right]$. By (3.6) and Lemma 2 , the last identity gives

$$
\left[\left(1+a^{p-1}\right)^{n}, y\right]=0 \text { for all } y \in R
$$

Combining (3.5) and (3.7) yields $0=\left[\left(1+{ }^{p-1}\right)^{n}, y\right]=\left[1+n a^{p-1}, y\right]=n\left[a^{p-1}, y\right]$. By $Q(n)$, the last identity gives $\left[a^{p-i}, y\right]=0$ for all $y \in R$. which is a contradiction to the minimality of $p$. Therefore $p=1$, and hence (3.4) holds.

Combining Lemma 7 and Lemma 8 , one gets

LEMMA 9. Let $n>1, m$, and s be fixed non-negativo integers, and let $R$ be a ring with unity 1 satisfying the polynomial identity (3.3). If, further, $R$ has $Q(n)$, then

$$
C(R) \subseteq Z(R)
$$

REMARK 1. In view of (3.8), it is guaranteed that $[x,[x, y]]=0$ for all $x, y \in R$. Thus (2.1) holds for each pair of elenents.,$y$ in a ring $R$ that satisfies the hypothesis of Lemma 9 .

Similarly, we have

LEMMA 10. Let $m>1, n$, and $t$ be fixed non-negative integers, and let $R$ be a ring with unity 1 satisfying the polynomial identity

$$
\left[x^{n}, y\right]=\left[x, y^{\prime \prime}\right] x^{\prime} \text { for all } x, y \in R .
$$

If, further, $R$ has $Q(m)$, then

$$
C(R) \subseteq Z(R)
$$

LEMMA 11. Let $m \geq 1, n \geq 1, s$ and $t$ be fixed non-negative integers, and let $R$ be a ring with unity 1 satisfying the polynomial identity (1.1). If, further, $R$ has

$$
[x,[x, y]]=0 \text { for all } x, y \in R,
$$

then the properties $Q(n)$ and $Q(m)$ are equivalent.

PROOF. In view of (3.11), and Lemma 1, the polynomial identity (1.1) becomes

$$
n y^{s}[x, y] x^{n-1}=m y^{m-1}[x, y] x^{t} \text { for all } x, y \in R
$$

Let $R$ have the property $Q(n)$. If $m[x, y]=0$ for all $x, y \in R$, then (3.12) gives $n y^{s}[x, y] x^{n-1}=$ 0 . By Lemma 2, we obtain $n[x, y]=0$ for all $x, y \in R$. Thus $Q(n)$ implies that $[x, y]=0$ for all $x, y \in R$. Therefore, $Q(n)$ implies $Q(m)$.

Similarly, one can prove that $Q(m)$ implies $Q(n)$.

THEOREM 2. Let $n>1, m, s$, and $t$ be fixed non-negative integers such that $s \neq m-1$, or $t \neq n-1$, and let $R$ be a ring with unity 1 satisfying the polynomial identity (1.1). Suppose that

(i) $R$ has $Q(n)$,

(ii) $N(R) \subseteq Z(R)$ for $t>0$,

(iii) $N^{\prime}(R) \subseteq Z(R)$ for $t>0$.

Then $R$ is commutative.

PROOF. Since we know that $C(R) \subseteq Z(R)$ by Lemma 9, and (ii), we shall routinely use equation (2.1) without explicit mention.

Now, if $m=0$, then (1.1) becomes $y^{y}\left[x^{n}, y\right]=0$, and by Lemma 2 , we have $\left[x^{n}, y\right]=0$, and hence $n x^{n-1}[x, y]=0$. Again by Lemma 2 along with $Q(n)$, we get $[x, y]=0$ for all $x, y \in R$. Thus $R$ is commutative.

Next, let $m \geq 1$, and then replace $x$ (resp. $y$ ) by $2 x$ (resp. $2 y)$ in (1.1) to get $2^{n} y^{s}\left[x^{n}, y\right]=$ $2^{t+1}\left[x, y^{m}\right] x^{t}$ or $2^{s+1} y^{s}\left[x^{n}, y\right]=2^{m}\left[x, y^{m}\right] x^{l}$. Combining this with (1.1) yields $\left|2^{n}-2^{t+1}\right|$ $\left[x, y^{m}\right] x^{t}=0(n \neq t+1)$ or $\left|2^{m}-2^{s+1}\right|\left[x, y^{m}\right] x^{t}=0(m \neq s+1)$. In view of Lemma 2 and Lemma 1 , we get $\left|2^{n}-2^{t+1}\right| m[x, y]=0$ or $\left|2^{m}-2^{s+1}\right| m[x, y]=0$. Let $k_{1}=\left|2^{n}-2^{t+1}\right| m$ and $k_{2}=\left|2^{m}-2^{s+1}\right| m$. If $k=k_{1}$ or $k_{2}$, then $k>1$. Hence $k[x, y]=0$ for all $x, y \in R$. Thus $\left[x^{k}, y\right]=k x^{k-1}[x, y]=0$ for all $x, y \in R$. Therefore, 


$$
x^{h} \in Z(R) \text { for all } x \in R .
$$

Now, (1.1) becomes $n y^{s}[x, y] \cdot x^{n-1}=\left[x \cdot y^{\prime \prime}\right] r^{\prime}$. Replace $x$ ly $x^{n}$ in the last identity to get

$$
n y^{*}\left[x^{\prime \prime} \cdot y\right] \cdot x^{n(n-1)}=\left[x^{n} \cdot y^{m}\right] \cdot x^{\prime \prime t} \text {. }
$$

Hence $n y^{s}\left[x^{n}, y\right] x^{n(n-1)}=n\left[x, y^{m}\right] x^{n t+n-1}$. Thus $n y^{s}\left[x^{n}, y\right] x^{n(n-1)}=n y^{s}\left[x^{n}, y\right] x^{(n-1)(t+1)}$. So $n\left[x^{n}, y\right] x^{(n-1)(t+1)}\left(1-x^{(n-1)(n-t-1)}\right)=0$. By Lemma 3 ,

$$
n\left[x^{n}, y\right] x^{(n-1)(t+1)}\left(1-x^{\Lambda(n-1)(n-t-1)}\right)=0 \text { for all } x, y \in R .
$$

It is well-known that $R$ is isomorphic to a subdirect sum of subdirectly irreducible rings $R_{\imath}\left(\imath \in I\right.$, the index set). Each $R_{\imath}$ has 1 , and satisfies (1.1), (3.1), (3.13), and (3.14), but $R_{\imath}$ does not necessarily have $Q(n)$.

Let $S$ be the intersection of all non-zero idleals of $R_{t}$. Then $S \neq 0$. Thus if $d$ is a central zero-divisor, then $S d=0$, since the annihilator of $d$ is a two-sided ideal and must therefore contain $S$.

If $t=0$, then by (3.14),

$$
n\left[x^{n}, y\right] x^{n-1}\left(1-x^{k(n-1)^{2}}\right)=0 \text { for all } x, y \in R_{\imath} .
$$

Let $a \in N^{\prime}\left(R_{2}\right)$. Then $(3.15)$ yields $n\left[a^{n}, y\right] a^{n-1}\left(1-a^{k(n-1)^{2}}\right)=0$. If $n\left[a^{n}, y\right] a^{n-1} \neq 0$, then $a^{k(n-1)^{2}}$ and $1-a^{k(n-1)^{2}}$ are central \%eno-divisors. Hence $0=S\left(1-a^{(1 n-1)^{2}}\right)=S$ which gives a contradiction. Thus

$$
n\left[a^{n}, y\right] a^{n-1}=0 \text { for all } y \in R_{\imath} .
$$

Combining (3.1) and (3.16) gives $y^{s^{\prime}}\left[a^{n^{2}}, y\right]=\left[a, y^{m^{2}}\right]$. So $\left[a, y^{m^{2}}\right]=n y^{s^{\prime}}\left[a^{n}, y\right] a^{n(n-1)}$. Thus

$$
\left[a, y^{m^{2}}\right]=0 \text { for all } y \in R_{\imath} \text {. }
$$

By (3.17), and (iii), for $t \geq 0$,

$$
\left[a, y^{m^{2}}\right]=0 \text { for all } y \in R_{\imath} .
$$

Next, let $c \in Z\left(R_{z}\right)$. Replace $x$ by $c x$ in (1.1) and apply Lemma 2 to obtain $\left(c^{n}-\right.$ $\left.c^{t+1}\right)\left[x, y^{m}\right]=0$ and $\left(c^{t+1}-c^{n}\right)\left[x, y^{m}\right]=0$ for all $x, y \in R_{\imath}$. In particular, by (3.13), we see that

$$
\left(x^{k n}-x^{k(t+1)}\right)\left[x, y^{m}\right]=0 \text { for all } x, y \in R_{\imath},
$$

and

$$
\left(x^{k(t+1)}-x^{k n}\right)\left[x, y^{m}\right]=0 \text { for all } x, y \in R_{r} .
$$

Similarly, we obtain

$$
\left(x^{k m}-x^{h(s+1)}\right)\left[x, y^{m}\right]=0 \text { for all } x, y \in R_{\imath},
$$

and

$$
\left(x^{k(s+1)}-x^{k m}\right)\left[x, y^{\prime \prime \prime}\right]=0 \text { for all } x, y \in R_{\imath} .
$$

If $\left[x, y^{m^{2}}\right] \neq 0$, then $\left[x, y^{m}\right] \neq 0$ for all $x, y \in R_{\imath}$. Now, let $n-t>1$. Then (3.18) gives that $x^{k n}-x^{k(t+1)}$ is a zero-divisor. Therefor e, $x^{h(n-t-1)+1}-x$ is also a zero-divisor. Similarly, if $m-s>1$, then $x^{k(m-s-1)+1}-x$ is also a zero-divisor. Let $q_{1}=k(n-t-1)+1$ or $k(m-s-1)+1$. Then $q_{1}>1$, and $(3.17)^{\prime}$ implies that

$$
\left[x^{q_{1}}-x, y^{m^{2}}\right]=0 \text { for all } x, y \in R_{2} .
$$

Since each $R_{i}(i \in I)$ satisfies (3.20), the original ring $R$ also satisfies (3.20). Thus $m^{2}\left[x^{q_{1}}-x, y\right] y^{m^{2}-1}=0$. By Lemma $2, m^{2}\left[x^{q_{1}}-x, y\right]=0$ for all $x, y \in R$. But $C(R) \subseteq Z(R)$, and $R$ has $Q(n)$. So $R$ has $Q(m)$ by Lemma 11 . Hence $\left[x^{q_{1}}-x, y\right]=0$ for all $x, y \in R$. Therefore, $R$ is commutative by Theorem $\mathrm{H}$.

Similarly, if $n-t<1$ or $m-s<1$, we can see that $\left[x^{q_{2}}-x, y\right]=0$ for all $x, y \in R$, where $q_{2}=k(t-n+1)+1$ or $k(s-m+1)+1$, and $q_{2}>1$. Again $R$ is commutative by Theorem $\mathrm{H}$. 
If $\left[x, y^{m^{2}}\right]=0$, then clearly $\left[x^{q}-x, y^{m^{2}}\right]=0$ for all positive integers $q$ and $x, y \in R_{\imath}$. Following the same argument as above, we see that $\left[x^{q}-x, y\right]=0$ for all $x, y \in R$. Therefore, $R$ is also commutative by Theorem $\mathrm{H}$.

REMARK 2. It should be noted that in Theorem 2, if $t=0$, and $m=1$, then (1.1) gives

$$
y^{s}\left[x^{n}, y\right]=[x, y] \text { for all } x, y \in R,
$$

and $R$ is commutative even if $R$ is not assumed to be with unity 1 . Indeed, the $s=0$ case in (3.21) is an old theorem of Herstein (Theorem H). If $s \geq 1$ in (3.21), then $R$ is a $(\mathbf{Z}, \bar{\beta})$-ring in the sense of Streb ([10]). Hence $R$ is commutative. Also, if $R$ satisfies (3.21), then $R$ is commutative by [11, Theorem].

THEOREM 3. Let $m>1, n, s$, and $t$ be fixed non-negative integers such that $s \neq m-1$, or $t \neq n-1$, and let $R$ be a ring with unity 1 satisfying the polynomial identity (1.1). Suppose that

(i) $R$ has $Q(m)$,

(ii) $N(R) \subseteq Z(R)$ for $s>0$,

(iii) $N^{\prime}(R) \subseteq Z(R)$ for $s>0$.

Then $R$ is commutative.

PROOF. By Lemma 10 and (ii), $C(R) \subseteq Z(R)$ for $s \geq 0$. Interchange $x$ and $y$ in (1.1) to get $\left[x^{m}, y\right] y^{t}=x^{s}\left[x, y^{n}\right]$, and thus $y^{t}\left[x^{m}, y\right]=\left[x, y^{n}\right] x^{s}$ for all $x, y \in R$. Therefore, $R$ is commutative by Theorem 1 .

If, we replace in Theorem 1 , the condition " $R$ has $Q(n)$ " by " $m$ and $n$ are relatively prime integers," then $R$ is still commutative with some restrictions on $s$, and $t$.

THEOREM 4. Let $m>1$, and $n>1$ be relatively prime integers, and let $s$, and $t$ be fixed non-negative integers such that $s \neq m-1$ or $t \neq n-1$. Suppose that $R$ is a ring with unity 1 which satisfies (1.1). If further, $N(R) \subseteq Z(R)$, and $N^{\prime}(R) \subseteq Z(R)$, for the cases $s>0, t=0 ; s=0, t>0$; and $s>0, t>0$, then $R$ is commutative.

PROOF. As is well-known $R$ is isomorphic to a subdirect sum of subdirectly irreducible rings $R_{z}(i \in I)$, each of which as a homomorphic image of $R$ satisfies the property placed on $R$. Thus $R$ can be assumed to be subdirectly irreducible.

If $s=t=0$, then (1.1) becomes $\left[x^{n}, y\right]=\left[x, y^{m}\right]$. Let $a \in N(R)$. Following the same argument as in the proof of Lemma 8 , we get $n\left[a^{p-1}, y\right]=0$ and $m\left[a^{p-1}, y\right]=0$ for all $x, y \in R$. Since $m$ and $n$ are relatively prime, by Lemma 6 , we get $\left[a^{p-1}, y\right]=0$. Hence, $[a, y]=0$ for all $y \in R$. Thus $N(R) \subseteq Z(R)$, when $s=t=0$ in (1.1). Therefore, By Lemma 7 , and the hypothesis,

$$
C(R) \subseteq N(R) \subseteq Z(R)
$$

for all $s$, and $t$.

We notice that the proof of (3.13) also works in the present situation, so there exists $k$, as in the proof of Theorem 2, such that

$$
x^{k} \in Z(R) \text { for all } x \in R .
$$

Furthermore, if $s=t=0$ in (1.1), and $u \in N^{\prime}(R)$, then we obtain, as in the proof of Theorem $2,\left[u, y^{m^{2}}\right]=0$ and $\left[u, y^{n^{2}}\right]=0$ for all $y \in R$. Thus Lemma 5 yields $u \in Z(R)$. Therefore, for all $s$, and $t$,

$$
N^{\prime}(R) \subseteq Z(R)
$$

If $c \in Z(R)$, then as in the proof of Theorem 2, we see that $n\left(c^{n}-c^{t+1}\right)[x, y]=0$ for all $x, y \in R$, and a variation of the argument yields $m\left(c^{n}-c^{t+1}\right)[x, y]=0$ for all $x, y \in R$. By Lemma 6 , we get $\left(c^{n}-c^{t+1}\right)[x, y]=0$ for all $x, y \in R$, and similarly, $\left(c^{t+1}-c^{n}\right)[x, y]=$ 0 for all $x, y \in R$. Thus $\left(x^{k n}-x^{k(t+1)}\right)[x, y]=0$, for $n-t>1$, and $\left(x^{k(t+1)}-x^{k n}\right)[x, y]=0$ for $n-t<1$ for all $x, y \in R$. By the same argument. $\left(x^{k m}-x^{k(s+1)}\right)[x, y]=0$ for $m-s>1$ and $\left(x^{k(s+1)}-c^{k m}\right)[x, y]=0$ for $m-s<1$ for all $x, y \in R$.

Hence, we complete the proof by arguing as in the proof of Theorem 2, to prove that $x^{q_{1}}-x \in Z(R)$, or $x^{q_{2}}-x \in Z(R)$, for all $x \in R$, where $q_{1}, q_{2}$ are as in the proof of Theorem

2. Therefore, $R$ is commutative by Theorem $\mathrm{H}$. 
We conclude with the following special cases of Theorem 2 .

COROLLARY 1 ([3, Theorem 5]). Let $R$ be a ring with unity 1 , and $n>1$ be a fixed integer. If $R$ is $n$-torsion free and satisfies the identity $\left[x^{n}, y\right]=\left[x, y^{n}\right]$ for all $x, y \in R$, then $R$ is commutative.

By using Proposition 1 of [1], we can assume that any s-unital ring is a ring with unity 1.

COROLLARY $2([2$, Lemma $2(2)])$. Let $n$ be a fixed positive integer, and let $R$ be an s-unital ring in which every commutator is $n$-torsion free. If $R$ satisfies the polynomial identity $\left[x^{n}, y\right]=0$ for all $x, y \in R$, then $R$ is commutative.

COROLLARY 3 ([4, Theorem 2]). Let $n \geq m \geq 1$ be fixed integers such that $m n>1$, and let $R$ be an s-unital ring. Suppose every commutator in $R$ is $m$ !-torsion free. Further, if $R$ satisfies the polynomial identity $\left[x^{n}, y\right]=\left[x, y^{m}\right]$ for all $x, y \in R$, then $R$ is commutative.

REMARK 3. Let $R$ be the subring of the ring of all $3 \times 3$ matrices over $G F(4)$ such that

$$
R=\left\{\left(\begin{array}{ccc}
a & b & c \\
0 & a^{2} & 0 \\
0 & 0 & a
\end{array}\right) \mid a, b, c \in G F(4)\right\}
$$

It is readily verified that $R$ is non-commutative local ring with unity, and characteristic 2 . Also $R$ is 3 -torsion free. Further, $R$ satisfies $y\left[x^{3}, y\right]=\left[x, y^{2}\right] x^{2}$. We notice that for each element $x \in R$, either $x^{2}=x^{7}=0$ or $x^{7}=x$ (see [12]). Hence, $R$ satisfies $y^{7}\left[x^{3}, y\right]=\left[x, y^{2}\right] x^{8}$, for which $s \neq m-1$, and $t \neq n-1$. Further, the nilpotent elements, and the zero-divisors of $R$ are not in the center of $R$.

ACKNOWLEDGMENT. I would like to express my gratitude and indebtedness to the referee for his helpful suggestions and valuable comments. Also, I extend my thanks to Professor V. Perić (University of Sarajevo, Yugoslavia), and Professor T. P. Kezlan (University of Missouri, Kansas City, U. S .A) for their helpful suggestions.

\section{REFERENCES}

1. HIRANO, Y., KOBAYASHI, Y., AND TOMINAGA, H., Some Polynomial Identities and Commutativity of s-Unital Rings, Math. J. Okayama Univ., 24 (1982), 7-13.

2. ABU-KHUZAM, H., TOMINAGA, H., AND YAQUB, A., Commutativity Theorems for s-Unital Rings Satisfying Polynomial Identities, Math. J. Okayama Univ., 22 (1980), 111-114.

3. BELL, H. E., On the Power Map and Ring Commutativity, Canad. Math. Bull., 21 (1978), 399-404.

4. PSOMOPOUlOS, E., TOMINAGA, H., And YAQUB, A., Some Commutativity Theorems for n-Torsion Free Rings, Math. J. Okayama Univ., 23 (1981), 37-39.

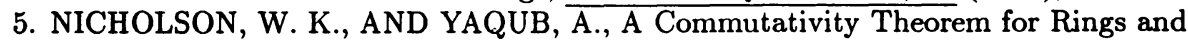
Groups, Canad. Math. Bull., 22 (1979), 419-423.

6. QUADRI, M. A., AND KHAN, M. A., A Commutativity Theorem for Left s-Unital Rings, Bull. Inst. Math. Acad. Sinica, 15 (1987), 323-327.

7. KEZLAN, T. P., A Note On Commutativity of Semiprime PI-Rings, Math. Japon., 27 (1982), 267-268.

8. BELL, H.E., On Rings With Commuting Powers, Math. Japon., 24 (1979), 473-478.

9. HERSTEIN, I.N., A Generalization of a Theorem of Jacobson, Amer. J. Math., 73 (1951), 756-762.

10. STREB, W., Über Einen Satz Von Herstein und Nakayama, Rend. Sem. Mat. Univ. Padova, 64 (1981), 159-171.

11. KEZLAN, T. P., On Identities Which are Equivalent With Commutativity, Math. Japon., 29 (1984), 135-139.

12. MOORE, H.G., Generalized $n$-Like Rings and Commutativity, Canad. Math. Bull., 23 (1980), 449-452. 


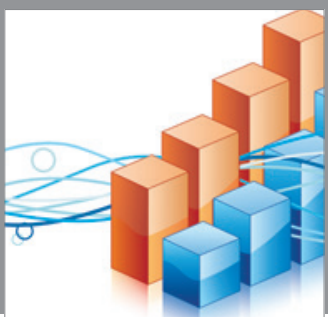

Advances in

Operations Research

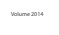

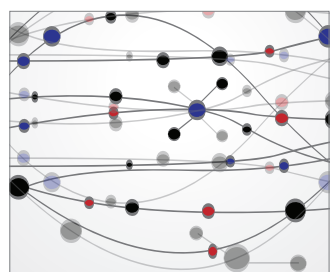

\section{The Scientific} World Journal
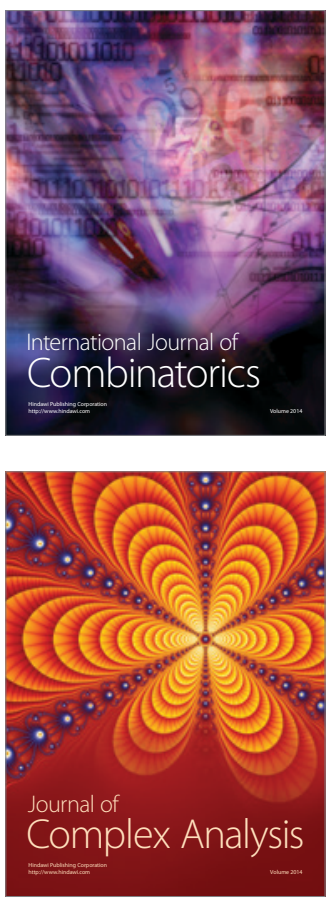

International Journal of

Mathematics and

Mathematical

Sciences
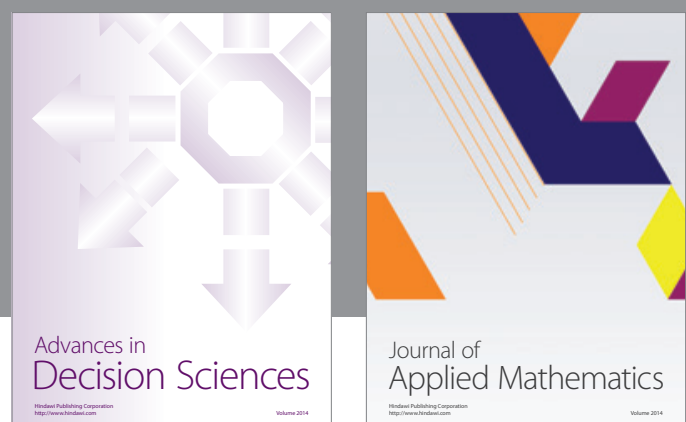

Journal of

Applied Mathematics
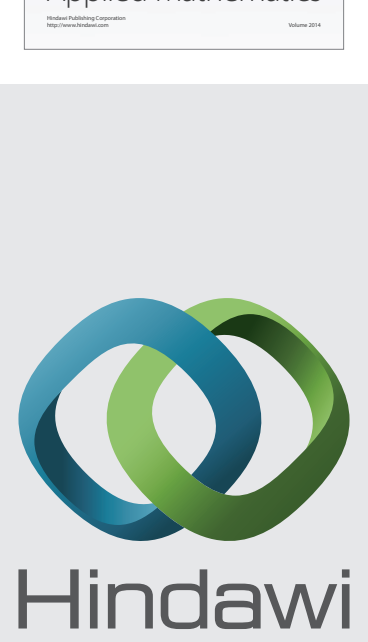

Submit your manuscripts at http://www.hindawi.com
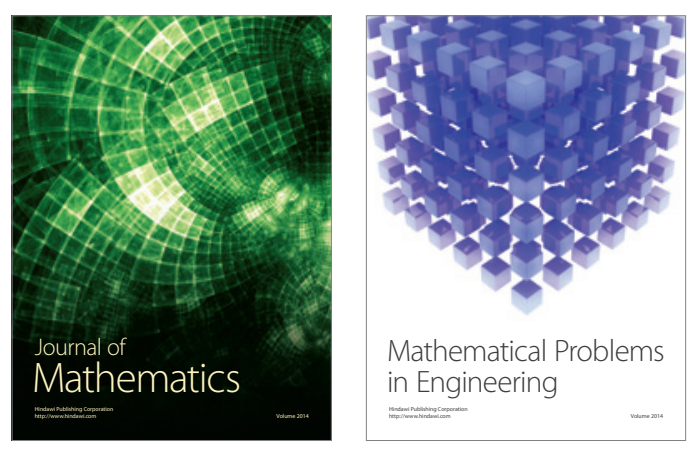

Mathematical Problems in Engineering
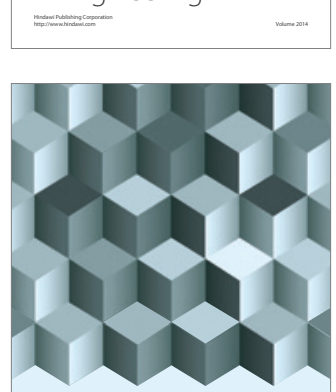

Journal of

Function Spaces
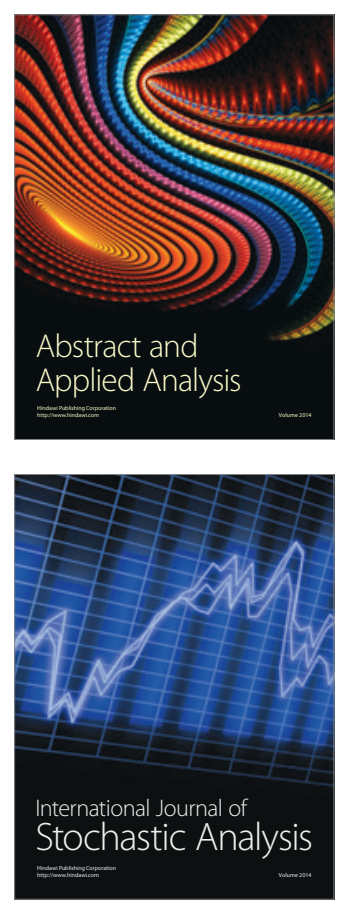

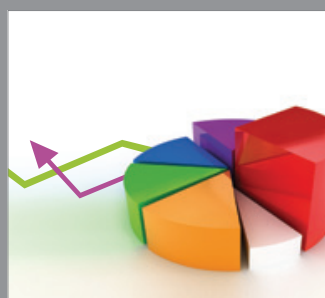

ournal of

Probability and Statistics

Promensencen
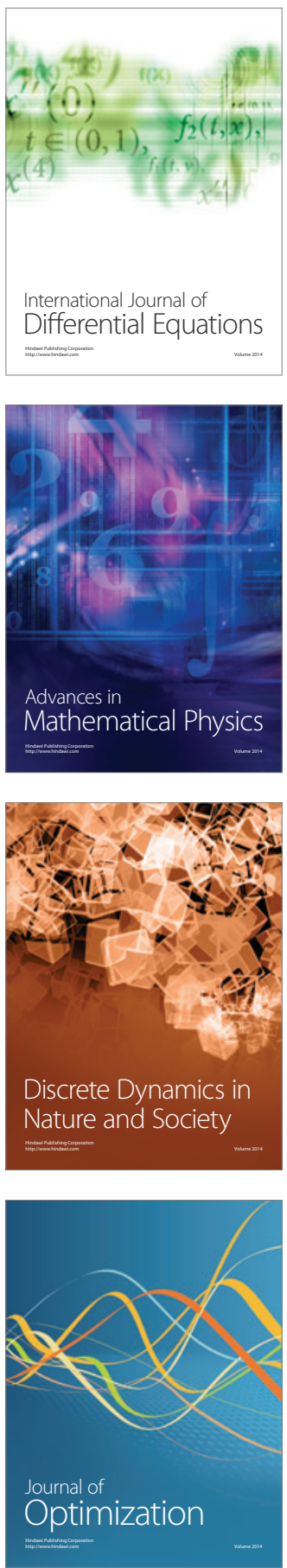